\section{Risco cardiovascular e prática de atividade física em adultos de Muzambinho/MG: influência do gênero e da idade}

\section{Cardiovascular risk and physical activity in adults from Muzambinho/MG: influence of gender and age}

\author{
João Paulo dos Anjos Souza Barbosa ${ }^{1}$ \\ Teresa Bartholomeu ${ }^{1}$ \\ Januária Andrea Souza Rezende ${ }^{2,3}$ \\ Luciano Basso ${ }^{1}$ \\ Jorge Alberto Oliveira \\ Go Tani \\ António Prista ${ }^{4}$ \\ José António Ribeiro Maia ${ }^{5}$ \\ Cláudia Lúcia de Moraes Forjaz ${ }^{1}$
}

\section{Resumo}

O objetivo deste estudo foi verificar a prevalência de fatores de risco cardiovascular (FRC) e da prática de atividade física (AF) em diferentes domínios em adultos de Muzambinho-MG, avaliando a influência do gênero e da idade. Para tanto, o peso corporal, a estatura, a glicemia, a colesterolemia, a pressão arterial e a prática de $\mathrm{AF}$ (ocupacional, deslocamento e lazer) foram avaliados em 223 adultos desta cidade (37,2 $\pm 6,3$ anos, $57 \%$ mulheres). As comparações entre gêneros (homens e mulheres) e idades (mediana do grupo - > e $\leq 36,9$ anos) foram feitas pelo teste Qui-quadrado. $\mathrm{P}<0,05$ foi aceito como significante. As prevalências dos FRC foram elevadas para excesso de peso $(54,6 \%)$ e obesidade central $(30,4 \%)$. Contrariamente, apenas $4,1 \%$ dos indivíduos foram considerados insuficientemente ativos ou inativos, sendo que 52,2\% relataram praticar alguma AF de deslocamento, $89,3 \%$ ocupacional e $34,8 \%$ de lazer. Os homens apresentaram maior prevalência de sobrepeso, colesterol alto e prática de alguma AF de lazer. As mulheres apresentaram maior prevalência de obesidade, obesidade central e prática de alguma AF de deslocamento. Os indivíduos com mais de 36,9 anos apresentaram maior prevalência de colesterol elevado e hipertensão, menor prevalência de prática de alguma AF ocupacional e maior prevalência de $\mathrm{AF}$ suficiente de lazer. Conclui-se, portanto, que nos adultos residentes da cidade de Muzambinho, a prevalência dos FRC referentes à gordura corporal foi elevada apesar da alta prevalência de $\mathrm{AF}$ total suficiente. A prevalência dos FRC e da prática de AF nos diferentes domínios diferiram entre gêneros e idades.

\section{Palavras-chave}

Fatores de risco; Obesidade; Hipertensão arterial; Diabetes mellitus; Hipercolesterolemia; Atividade motora.

\begin{abstract}
The aim of this study was to verify the prevalence of cardiovascular risk factors (CRF) and different domains of physical activity (PA) in adults from Muzambinho-MG, verifying the influence of gender and age. For that, weight, height, glycemia, cholesterolemia, blood pressure and PA (occupational, commuting, and leisure time) were evaluated in 223 adults from this city (37.2 \pm 6.3 years, 57\% of women). Comparisons between genders (men and women) and age groups (sample median- $>$ and $\leq$ 36.9 years) were made by chi-square test. $P<0.05$ was accepted as significant. CRF prevalences were high for body weight excess (54.6\%) and central obesity (30.4\%). On contrary, only $4.1 \%$ of the subjects were classified as insufficiently active or inactive, whereas $52.2 \%$ reported the practice of commuting $P A$, 89.3\% occupational PA and $34.8 \%$ leisure time PA. Men presented a higher prevalence of overweight, high cholesterolemia and practice of leisure $P A$. Women presented higher prevalence of obesity, high waist circumference and practice of commuting $P A$. Subjects over 36.9 years presented higher prevalence of high cholesterolemia and hypertension, lower prevalence of practice of occupational $P A$ and higher prevalence of sufficient leisure PA. It is concluded, therefore, that in adults living in the city of Muzambinho, the prevalence of $C R F$ related to body fat was high despite of high levels of sufficient total $P A$. The prevalences of CRF and different domains $P A$ practice differed between the genders and changed with age.
\end{abstract}

\section{Keywords}

Risk factors; Obesity; Hypertension; Diabetes mellitus; Hypercholesterolemia; Motor activity.
Rev Bras Ativ Fis Saúde p. 242-252 DOl:

http://dx.doi.org/10.12820/rbafs.v.18n2p242

1 Escola de Educação Física e Esporte, Universidade de São Paulo, São Paulo, Brasil. 2 Escola Superior de Educação Física de Muzambinho, Minas Gerais, Brasil.

3 Instituto Federal de Educação, Ciências e Tecnologia do Sul de Minas Gerais - Campus Muzambinho, Minas Gerais, Brasil.

4 Faculdade de Educação Física e Desporto, Universidade Pedagógica, Maputo, Moçambique.

5 CIFID, Faculdade de Desporto, Universidade do Porto, Portugal. 


\section{INTRODUÇÃO}

A principal causa de morte em adultos e idosos no Brasil ${ }^{1}$ e no mundo ${ }^{2}$ deve-se às doenças cardiovasculares (DCV). Obesidade, diabetes, hipercolesterolemia e hipertensão arterial são importantes fatores de risco cardiovascular $(\mathrm{FRC})^{2} \mathrm{e}$, desta forma, o controle destes fatores tem grande importância na redução da morbimortalidade cardiovascular. Por outro lado, a prática de atividade física (AF) é um fator protetor no desenvolvimento das doenças crônicas e, principalmente, das DCV $\mathrm{D}^{3}$. Assim, esta prática faz parte do Plano de Ações Estratégicas para Enfrentamento das Doenças Crônicas Não Transmissíveis no Brasil e é considerada uma pedra angular para este objetivo ${ }^{4}$.

Entretanto, para se conhecer a real proporção do problema no país, torna-se necessário investigar a prevalência dos FRC na população. Segundo dados de inquéritos telefônicos do Ministério da Saúde (VIGITEL) ${ }^{5,6}$, as prevalências dos FRC nos adultos brasileiros são de: $42 \%$ para o excesso de peso (somatória de obesidade e sobrepeso), $12 \%$ para a obesidade, $5,6 \%$ para o diabetes, $16,9 \%$ para a dislipidemia e $22,7 \%$ para a hipertensão arterial. Contudo, estas prevalências variam nos diferentes estados ${ }^{6}$ e em cidades específicas ${ }^{7,8}$. Por exemplo, a prevalência de obesidade variou de 39,8 a 55,4\% entre as capitais brasileiras ${ }^{6}$ e, em cidades de São Paulo, esta prevalência variou de 14,9 a $15,7 \%{ }^{6,9}$.

Da mesma forma, a prevalência de prática de AF nos diferentes domínios também oscila na população adulta brasileira. No VIGITEL, a prevalência média de $\mathrm{AF}$ suficiente de lazer foi de $30,3 \%$ e considerando a $\mathrm{AF}$ de deslocamento foi de $17,0 \%{ }^{6}$. Porém, considerando-se os diferentes estados, a prevalência de AF suficiente de lazer variou de 26,3 a 41,4\% nas diferentes capitais ${ }^{6}$. Estes índices também variam entre cidades de diferentes tamanhos dentro do mesmo estado. No estado de São Paulo, a prevalência de AF suficiente de lazer foi de 27,5\% na capital $(\approx 11.000 .000 \text { de habitantes })^{6}$, de $32,2 \%$ em Bauru $(\approx 350.000 \text { habitantes })^{10}$ e de $42,1 \%$ em Botucatu ( $\approx 130.000$ habitantes $)^{9}$.

A grande variação das prevalências dos FRC e na prática de AF entre diferentes cidades brasileiras sugere que aspectos ambientais podem influenciá-las. Neste sentido, as prevalências dos FRC foram descritas, principalmente, em capitais e cidades de grande e médio porte ${ }^{6-8}$, de modo que as altas prevalências têm sido atribuídas às modificações do estilo de vida advindas do desenvolvimento urbano $^{2}$. Entretanto, grande parte das cidades brasileiras ainda apresenta pequeno porte ${ }^{11}$, nível de desenvolvimento urbano pequeno, e hábitos de vida tradicionais, sendo importante avaliar a prevalência dos FRC nessas cidades para uma compreensão mais abrangente do quadro nacional. Por estas características, Muzambinho-MG, foi escolhida como cidade alvo deste estudo. Esta cidade localiza-se na região cafeeira do sul de Minas Gerais, possui uma extensão territorial de $410 \mathrm{~km}^{2}$ e uma população de $\approx 21.000$ habitantes $^{12}$.

Em um estudo anterior ${ }^{13}$ investigamos a prevalência dos FRC nas crianças e adolescentes de Muzambinho e observamos, da mesma forma que em cidades de grande porte, prevalências elevadas (sobrepeso $=18,8 \%$, diabetes $=4,5 \%$, hipercolesterolemia $=14,5 \%$ e hipertensão $=10,7 \%$ ). No entanto, contrariamente aos estudos realizados em outras cidades, a prevalência de jovens suficientemente ativos na cidade de Muzambinho foi elevada. Estes dados mostram um padrão diferente do esperado por apresentar concomitância e não oposição entre altos níveis de AF e de presença de FRC. É possível que um quadro similar (alto risco cardiovascular concomitante a alta prática de AF) também seja observado em adultos de cidades 
pequenas, o que precisa ser investigado para nortear as atuações de saúde pública no combate às DCV. Desta forma, o objetivo deste estudo foi verificar a prevalência de $\mathrm{FRC}$ e da prática de $\mathrm{AF}$ em diferentes domínios em adultos residentes na cidade de Muzambinho-MG, avaliando a influência do gênero e da idade.

\section{MÉTODOS}

Foram convidados para este estudo, os pais das crianças que participaram do estudo anterior sobre risco cardiovascular de crianças e adolescentes de Muzambinho- $\mathrm{MG}^{13}$. Estas crianças foram selecionadas a partir das crianças com 10 anos de uma pesquisa que avaliou 25\% dos estudantes da rede pública de Muzambinho. Estas crianças pertenciam a 138 famílias nucleares, o que formou um universo amostral para este estudo de 276 adultos. $\mathrm{O}$ estudo foi aprovado pelo Comitê de Ética em Pesquisa da Escola de Educação Física e Esporte da USP e recebeu o registro CAAE-0033.0.342.000-07 no Conselho Nacional de Ética em Pesquisa. Os dados foram coletados no período de março de 2008 a agosto de 2009.

Os voluntários foram visitados em suas residências para a apresentação do projeto e a assinatura do termo de consentimento. Em seguida, foi agendada outra visita à residência para a medida de glicemia e colesterolemia de jejum. Num outro dia, os voluntários foram convocados a comparecerem à escola de seus filhos onde foi realizada a avaliação da presença de $\mathrm{FRC}$ conhecidos e da prática de $\mathrm{AF}$, além de serem realizadas medidas antropométricas e da pressão arterial. Após as coletas, os voluntários foram informados dos resultados das avaliações, receberam orientações de saúde e, quando necessário (FRC presentes e descontrolados), foram encaminhados para acompanhamento médico.

\section{Medidas}

O risco cardiovascular conhecido foi avaliado em forma de entrevista por um questionário elaborado com base nas sugestões do American College of Sports $M e-$ dicine $(A C S M)^{14}$. O gênero e a idade de cada voluntário foram anotados. Eles foram questionados sobre:

- Antecedentes pessoais de DCV: a) diagnóstico médico de DCV, b) cirurgias cardíacas prévias, c) acometimentos cardiovasculares prévios, e d) uso de medicamentos para DCV;

- Antecedentes pessoais de diabetes, dislipidemia ou hipertensão arterial: a) diagnóstico médico ou uso de medicamentos para o tratamento dessas doenças;

O peso e a estatura foram medidos numa balança com estadiômetro (Filizola, Brasil). A circunferência da cintura foi medida com uma fita métrica posicionada na altura da cicatriz umbilical. Foi calculado o índice de massa corporal (IMC) pelo quociente entre o peso (quilos) e o quadrado da estatura (metros).

A glicemia e a colesterolemia total foram medidas por monitores automáticos (Glicemia = Accu-Check, Advantage II, Roche, EUA e Colesterolemia - Accutrend Plus, Roche, Alemanha) através da punção digital, com os voluntários em jejum de pelo menos seis horas.

A pressão arterial foi medida pelo método auscultatório, utilizando-se um esfigmomanômetro aneróide calibrado (BIC, Brasil). A circunferência do braço foi medida e foi escolhido um manguito de tamanho adequado. A medida foi realizada após repouso de cinco minutos na posição sentada. Inicialmente, foram 
realizadas duas medidas consecutivas com intervalo de 30 segundos. Quando a diferença entre elas foi superior a quatro $\mathrm{mmHg}$, uma nova medida foi feita. $\mathrm{O}$ valor da pressão arterial de cada voluntário foi estabelecido pela média das duas ou das três medidas realizadas. As fases I e V dos sons de Korotkoff foram utilizadas para determinar, respectivamente, os valores da pressão arterial sistólica e diastólica.

Neste estudo, para se considerar os FRC presentes foram adotados os seguintes critérios:

- Obesidade: $\mathrm{IMC} \geq 30,0 \mathrm{~kg} / \mathrm{m}^{2} 15$;

- Sobrepeso: IMC entre 25,0 e 29,9 kg/m² 15;

- Excesso de peso: somatória da obesidade e sobrepeso;

- Obesidade central: circunferência da cintura $>102 \mathrm{~cm}$ para homens e 88 para mulheres ${ }^{15}$;

- Diabetes: antecedente pessoal de diabetes diagnosticado por médico, uso de medicamentos para tratamento da diabetes ou glicemia de jejum $>126 \mathrm{mg} / \mathrm{d}^{16}$;

- Colesterol elevado: antecedente pessoal de hipercolesterolemia diagnosticado por médico, uso de medicamentos para o controle de colesterol ou colesterolemia de jejum $>240 \mathrm{mg} / \mathrm{dl}^{17}$;

- Hipertensão arterial: antecedente pessoal de hipertensão diagnosticado por médico, uso de medicamentos anti-hipertensivos ou pressão arterial sistólica $>140 \mathrm{mmHg}$ e/ou pressão arterial diastólica $>90 \mathrm{mmHg}^{18}$;

A prática de $\mathrm{AF}$ foi avaliada por uma entrevista estruturada feita por um avaliador treinado e já aplicada em outros estudos ${ }^{13,19}$. Os voluntários foram questionados quanto às suas atividades ocupacionais (trabalho), de deslocamento e de lazer (tempo livre). Em relação à AF ocupacional, aos sujeitos foram questionados sobre sua ocupação e se ela implicava na realização de AF. Quanto ao deslocamento, eles foram questionados sobre como se deslocavam na cidade para a realização das suas tarefas diárias. Em relação à $\mathrm{AF}$ de lazer, os indivíduos foram questionados sobre as atividades realizadas no seu tempo livre. Para cada domínio de AF, foram questionadas a frequência semanal e a duração da prática. $\mathrm{O}$ volume semanal de prática em cada domínio foi calculado multiplicando-se a frequência semanal pela duração. O volume semanal de AF total foi calculado somando-se (sem a atribuição de pesos) os volumes semanais da prática ocupacional, de deslocamento e de lazer. Foram considerados com AF suficiente (ativos), os participantes que praticavam AF por mais de $150 \mathrm{~min} / \mathrm{semana}^{14}$. Esta classificação foi aplicada usando-se o volume de $\mathrm{AF}$ total e também os volumes de $\mathrm{AF}$ ocupacional, de deslocamento e de lazer, separadamente.

\section{Análise dos dados}

A tabulação dos dados foi feita com dupla entrada independente. Os dados foram processados no software Access (Office for Windows) e a comparação dos dois arquivos foi feita pelo programa EpiInfo (United States of Health and Human Services, 2005). Entradas discordantes foram checadas e corrigidas. $\mathrm{Na}$ análise descritiva, os dados são apresentados em média \pm desvio padrão e em distribuição de frequência. Para a divisão em faixas etárias, a amostra foi separada pela mediana da idade ( $\leq 36,9$ e $>36,9$ anos). As comparações de prevalências entre os gêneros e as faixas etárias foram realizadas através do teste Qui-quadrado. $\mathrm{P}<0,05$ foi aceito como significante. 


\section{RESULTADOS}

A amostra constou de 223 adultos com idade média de 37,2 $\pm 6,3$ anos (variando de 24 a 65 anos), sendo $57 \%$ mulheres. Entre as mulheres, a idade média foi de $35,4 \pm 5,8$ anos (variando de 24 a 49 anos) e entre os homens de 39,8 $\pm 7,2$ anos (variando de 27 a 65 anos). Entre os indivíduos abaixo e acima de 36,9 anos, 66\% e $47 \%$ eram mulheres, respectivamente.

Considerando-se a amostra total, entre os FRC estudados, o de maior prevalência foi o excesso de peso $(54,6 \%)$ e o menos prevalente foi o diabetes $(1,9 \%)$ (Figura 1A). Com relação à AF, 52,2\% dos adultos afirmaram praticar alguma AF de deslocamento, 89,3\% possuíam uma ocupação que envolvia $\mathrm{AF}$ e 34,8\% faziam alguma $\mathrm{AF}$ no seu tempo livre. Assim, 95,2\% dos adultos acumulavam 150 minutos ou mais por semana considerando-se em conjunto todos os domínios de AF, $87,4 \%$ tinham AF suficiente ocupacional, $32,4 \%$ de deslocamento e $16,9 \%$ de lazer (Figura 1B).

A

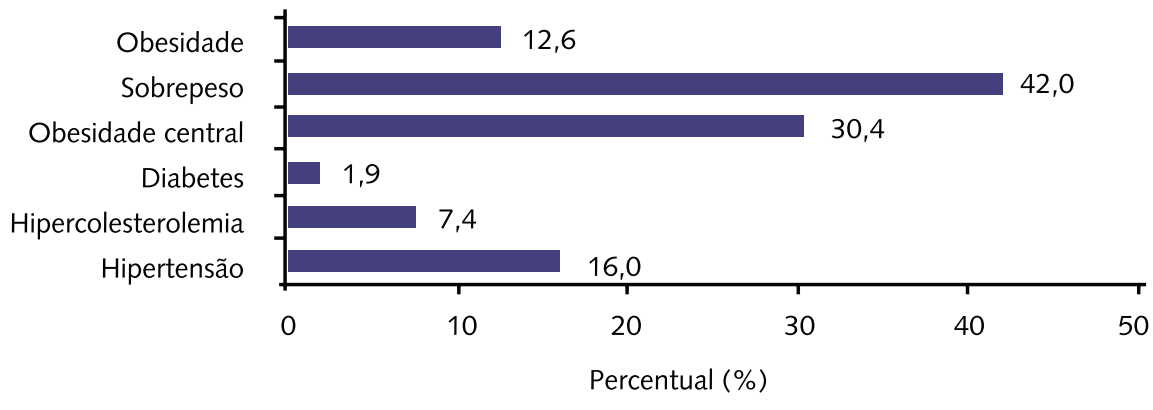

B

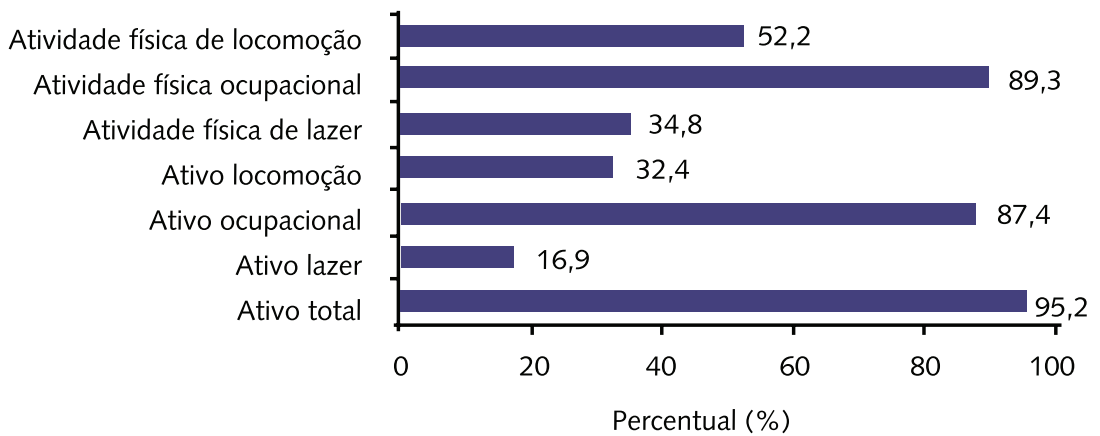

Figura 1 - Prevalências dos fatores de risco cardiovascular (A) e da prática de atividade física (B) dos adultos da cidade de Muzambinho-MG.

As comparações das prevalências dos FRC entre os gêneros e as faixas etárias estão apresentadas na Tabela 1 . O sobrepeso e a hipercolesterolemia foram mais prevalentes nos homens $(p<0,05)$, enquanto que a obesidade e a obesidade central foram mais prevalentes nas mulheres $(\mathrm{p}<0,05)$. A prevalência de hipertensão arterial $(p=0,882)$ e de diabetes $(p=0,175)$ não diferiu entre os gêneros. Considerandose as duas faixas etárias, os indivíduos acima de 36,9 anos apresentaram maiores prevalências de hipercolesterolemia e hipertensão arterial $(\mathrm{p}<0,05)$. Os demais fatores tiveram prevalências semelhantes entre aqueles acima e abaixo de 36,9 anos. 
Tabela 1 - Prevalências de fatores de risco cardiovascular, em função do gênero e de categorias de idade, em adultos de Muzambinho-MG.

\begin{tabular}{|c|c|c|c|c|c|c|c|c|c|c|}
\hline \multirow[t]{2}{*}{ Variáveis } & \multicolumn{2}{|c|}{ Masculino } & \multicolumn{2}{|c|}{ Feminino } & \multirow[t]{2}{*}{$\mathrm{p}$} & \multicolumn{2}{|c|}{$\leq 36,9$ anos } & \multicolumn{2}{|c|}{$>36,9$ anos } & \multirow[t]{2}{*}{$\mathrm{p}$} \\
\hline & $\mathrm{n}$ & $\%$ & $n$ & $\%$ & & $\mathrm{n}$ & $\%$ & $n$ & $\%$ & \\
\hline Obesidade & 6 & 7,1 & 20 & 16,4 & 0,046 & 14 & 13,3 & 12 & 11,8 & 0,734 \\
\hline Sobrepeso & 45 & 52,9 & 42 & 34,4 & 0,008 & 41 & 39,0 & 46 & 45,1 & 0,378 \\
\hline Obesidade Central & 8 & 9,4 & 55 & 45,1 & 0,000 & 38 & 36,2 & 25 & 24,5 & 0,068 \\
\hline Diabetes & 3 & 3,3 & 1 & 0,8 & 0,311 & 2 & 1,9 & 2 & 1,9 & 1,000 \\
\hline Hipercolesterolemia & 11 & 12,1 & 5 & 4,0 & 0,024 & 4 & 3,6 & 12 & 11,2 & 0,033 \\
\hline Hipertensão & 14 & 16,5 & 19 & 15,7 & 0,884 & 10 & 9,5 & 23 & 22,8 & 0,010 \\
\hline
\end{tabular}

As prevalências dos adultos que realizaram AF em cada domínio, divididas por gênero e faixa etária, estão apresentadas na Tabela 2. As mulheres apresentaram maior prevalência de prática de alguma $\mathrm{AF}$ de deslocamento e os homens de prática de alguma AF de lazer $(\mathrm{p}<0,05)$. A prevalência de AF suficiente de deslocamento foi maior nas mulheres, enquanto que a prevalência de $\mathrm{AF}$ suficiente nos outros domínios foi semelhante entre os sexos. Em relação à faixa etária, a prevalência de prática de alguma AF ocupacional foi menor nos sujeitos $>36,9$ anos, enquanto que a prevalência de prática de alguma $A F$ de lazer tendeu a ser maior $(\mathrm{p}=0,057)$ nestes sujeitos. Assim, a prevalência de AF suficiente de lazer foi maior no grupo $>36,9$ anos e a prevalência de AF total tendeu a ser menor neste grupo $(\mathrm{p}=0,056)$.

Tabela 2 - Percentual de sujeitos que relataram praticar alguma AF nos diferentes domínios e prevalência de AF suficiente em adultos de Muzambinho-MG, em função do gênero e categoria de idade.

\begin{tabular}{lcccccccccc}
\hline Variáveis & \multicolumn{2}{c}{ Masculino } & \multicolumn{2}{c}{ Feminino } & $\mathrm{p}$ & \multicolumn{2}{c}{$\leq 36,9$ anos } & \multicolumn{2}{c}{$>36,9$ anos } & $\mathrm{p}$ \\
\hline & $\mathrm{n}$ & $\%$ & $\mathrm{n}$ & $\%$ & & $\mathrm{n}$ & $\%$ & $\mathrm{n}$ & $\%$ & \\
\hline AF deslocamento & 33 & 38,8 & 75 & 61,5 & 0,001 & 60 & 57,1 & 48 & 47,1 & 0,146 \\
\hline AF ocupacional & 76 & 89,4 & 108 & 89,3 & 0,972 & 98 & 94,2 & 86 & 84,3 & 0,021 \\
\hline AF lazer & 43 & 50,6 & 29 & 23,8 & 0,000 & 30 & 28,6 & 42 & 41,2 & 0,057 \\
\hline At. deslocamento & 16 & 18,8 & 51 & 41,8 & 0,001 & 36 & 34,3 & 31 & 30,4 & 0,549 \\
\hline At. ocupacional & 75 & 88,2 & 106 & 86,9 & 0,773 & 96 & 91,4 & 85 & 83,3 & 0,079 \\
\hline At. lazer & 18 & 21,2 & 17 & 13,9 & 0,171 & 10 & 9,5 & 25 & 24,5 & 0,004 \\
\hline At. total & 81 & 95,3 & 116 & 95,1 & 0,944 & 103 & 98,1 & 94 & 92,2 & 0,056 \\
\hline
\end{tabular}

AF- Atividade física; At- Ativo

\section{DISCUSSÃO}

O principal achado deste estudo foi identificar nos adultos da cidade de Muzambinho, a coexistência de alta prevalência de FRC com níveis elevados de AF. Os FRC mais prevalentes foram os relacionados à gordura corporal: excesso de peso e obesidade central, enquanto que os domínios de prática de $\mathrm{AF}$ mais prevalentes foram a $\mathrm{AF}$ ocupacional e de deslocamento. Como achados secundários, verificouse que as prevalências dos FRC, bem como da prática de AF nos diferentes domínios variaram de acordo com o gênero e as categorias de idade: os homens apresentaram maior prevalência de sobrepeso e colesterol alto, enquanto praticavam mais AF de lazer. Por outro lado, as mulheres apresentaram mais obesidade e obesidade central, enquanto faziam mais $\mathrm{AF}$ de deslocamento. Com relação à idade, os adultos $>36,9$ anos apresentavam maior prevalência de colesterol alto e hipertensão, e praticavam mais AF de lazer e menos AF ocupacional que os $\leq 36,9$ anos.

A prevalência dos diversos $\mathrm{FRC}$ na população brasileira varia muito entre os estudos, o que tem sido atribuído às diferenças nas populações estudadas (cultu- 
ra, características genéticas, ambiente físico), nos indicadores de FRC utilizados, nas formas de avaliação dos FRC (questionário, medida direta) e nos pontos de corte utilizados $^{6-8}$. As prevalências encontradas na presente investigação para alguns FRC (diabetes=1,9\%, dislipidemia=7,4\% e hipertensão arterial=16,0\%) foram menores que as prevalências nacionais relatadas para estes fatores no VIGITEL (diabetes $=5,6 \%$, dislipidemia $=16,9 \%$ e hipertensão arterial $=22,7 \%)^{6}$. As diferenças entre estes resultados não pode ser atribuída aos critérios empregados para a obtenção dos dados, visto que o VIGITEL considerou apenas o relato da presença destes fatores, enquanto o presente estudo utilizou a informação autorrelatada e os valores de referência para os FRC de acordo com o $\mathrm{ACSM}^{14}$. Assim, as diferenças na obtenção dos dados explicariam uma prevalência mais alta no presente estudo e não mais baixa como foi observado. Esta baixa prevalência, entretanto, pode ser parcialmente atribuída à idade da população investigada, visto que a média de idade da amostra foi de 37 anos e havia apenas oito indivíduos com mais de 50 anos, enquanto que no VIGITEL foram avaliadas pessoas acima de 18 até mais de 65 anos. A prevalência destes FRC aumenta com a idade ${ }^{20} \mathrm{e}$, de fato, na amostra deste estudo, as prevalências de hipertensão arterial e de hipercolesterolemia foram maiores na faixa etária $>36,9$ anos. Outro aspecto que pode ser responsável pela baixa prevalência destes FRC é o fato da população estudada apresentar alta prevalência de $\mathrm{AF}$ total suficiente. Sabe-se que o nível de $\mathrm{AF}$ é inversamente relacionado à pressão arterial $^{21}$, glicemia ${ }^{22}$ e colesterolemia ${ }^{23}$, porém esta associação não permite estabelecer uma relação de causa e efeito nem uma direção para a possível causalidade.

Contrariamente ao observado nos FRC citados anteriormente, a prevalência de excesso de peso $(54,6 \%)$ foi bastante elevada nos adultos de Muzambinho, superando a prevalência relatada na população brasileira $(48,5 \%)^{6}$. Além disso, a prevalência de obesidade central $(30,4 \%)$ também foi alta. Estas prevalências, entretanto, foram semelhantes às observadas em outras cidades brasileiras de pequeno porte, como Luzerna, no interior de Santa Catarina, que tem $\approx 5.700$ habitantes e apresentou prevalências de $15,6 \%$ para a obesidade e $24,1 \%$ para a obesidade central $^{7}$ e também Baependi, também em Minas Gerais, uma cidade de $\approx 18.300$ habitantes que apresentou prevalência de $12,7 \%$ para a obesidade, $38,6 \%$ para o sobrepeso e $29,3 \%$ para a obesidade central ${ }^{8}$. A concomitância de alta prevalência de adultos ativos e de indivíduos com gordura corporal elevada parece inadequada perante o fato da prática regular de AF auxiliar no controle da gordura corporal ${ }^{24}$. Entretanto, é bastante conhecido que os fatores nutricionais têm efeito preponderante sobre este fator ${ }^{25} \mathrm{e}$, no presente estudo estes fatores não foram avaliados, sendo esta uma limitação do trabalho, que deve ser considerada em estudos futuros.

Apesar da alta prevalência de indivíduos ativos na cidade de Muzambinho, com mais de $95 \%$ dos adultos atingindo a classificação de suficientemente ativos, observa-se que, na maioria dos casos, este nível foi atingido devido à AF suficiente executada no trabalho. Um estudo com adultos de Bauru-SP também identificou um percentual elevado de AF suficiente neste domínio tanto nos homens (88\%) quanto nas mulheres $(96 \%)^{10}$. Entretanto, esta situação é diferente nas capitais brasileiras. Um estudo realizado em Goiânia identificou que apenas 21\% dos homens e $11 \%$ das mulheres são suficientemente ativos no trabalho ${ }^{26}$; no Rio de Janeiro, o percentual é menor com 3,6\% dos homens e apenas 0,3\% das mulheres com AF suficiente ocupacional ${ }^{27}$. De fato, nas cidades de pequeno porte as opções de trabalho ainda se relacionam às atividades com esforços físicos. Em Muzambinho, a maior parte dos adultos trabalha com atividades agrícolas (homens) ou domésticas (mulheres). Outra AF com prevalência acima dos valores observados 
em outros estudos foi a de deslocamento (52,2\%). No VIGITEL apenas 17\% dos brasileiros referiu $\mathrm{AF}$ suficiente de deslocamento ${ }^{6}$, com os valores variando de $11,8 \%$ a $21,1 \%{ }^{6}$. Esta diferença reflete, provavelmente, as diferenças no tamanho das cidades. Em Muzambinho, a ausência de um sistema público de transporte eficiente, somado à proximidade dos locais, estimula o indivíduo a deslocar-se caminhando ou pedalando para suas atividades diárias. É interessante observar que a prevalência de $\mathrm{AF}$ suficiente de lazer (16,9\%) em Muzambinho foi inferior à observada no VIGITEL (30,3\%), demonstrando que a alta AF desta população não ocorre no momento de lazer.

Analisando-se em conjunto as prevalências de FRC e AF na população de Muzambinho, observa-se um quadro contrário às expectativas. A presença de alta prevalência de $\mathrm{AF}$ total, devido às altas prevalências de $\mathrm{AF}$ suficientes de deslocamento e ocupacional, concomitante à alta prevalência dos FRC ligados à gordura corporal e baixa prevalência de outros FRC. Este quadro levanta a possibilidade de que diferentes domínios de $\mathrm{AF}$ apresentem efeitos distintos sobre os diferentes FRC, ou seja, os fatores relacionados à hipertensão, diabetes e dislipidemia poderiam responder ao aumento da $\mathrm{AF}$ independentemente de ser uma $\mathrm{AF}$ de lazer, ocupacional ou de deslocamento, enquanto que os fatores relacionados à gordura corporal poderiam responder mais efetivamente à prática de $\mathrm{AF}$ de lazer. Esta sugestão apresenta algum suporte científico numa meta-análise que demonstrou que o risco de mortalidade cardiovascular diminui com o aumento da $\mathrm{AF}$, mas esta redução é mais expressiva com a $\mathrm{AF}$ de lazer ${ }^{28}$. Além disso, o efeito da $\mathrm{AF}$ sobre os FRC parece apresentar uma relação dose-resposta ${ }^{14}$ e pode ter relação com a intensidade $^{14}$, o que talvez possa ser mais bem controlada nas AF de lazer. Entretanto, nenhum estudo, pelo nosso conhecimento, avaliou esta sugestão e, portanto, estudos futuros podem ser desenhados com este intuito. Outra possibilidade é que os fatores relacionados à gordura corporal sofram maior influência de outros fatores, como o nutricional e, por este motivo, sejam elevados apesar dos altos níveis de AF.

Considerando-se as diferenças observadas entre os gêneros, apesar da prevalência de excesso de peso ser igual entre homens e mulheres, a prevalência dos diferentes marcadores de gordura corporal alterada variou entre os sexos. A maior prevalência de obesidade central nas mulheres já foi relatada em outros estudos ${ }^{8}$ ${ }^{29}$, mas as diferenças entre os gêneros nos demais marcadores (excesso de peso, obesidade e sobrepeso) variam consideravelmente na literatura ${ }^{7,829}$. Da mesma forma, o efeito do aumento da idade na gordura corporal parece se caracterizar por aumento dos índices de excesso de peso e obesidade central ${ }^{25}$. Porém, neste estudo, este aumento não foi evidenciado, o que pode se dever à estreita faixa etária analisada ou aos altos níveis de alteração já observados na faixa etária mais baixa.

Em relação aos demais $\mathrm{FRC}$, a prevalência da hipertensão arterial parece ser maior nos homens que nas mulheres e aumentar com a idade ${ }^{18}$. Neste estudo, no entanto, a diferença entre os gêneros não foi observada, mas a prevalência foi maior na faixa etária acima dos 36,9 anos. Cabe ressaltar que nesta faixa etária, os valores de prevalência referentes à $\mathrm{AF}$ total suficiente tenderam a diminuir, o que pode se relacionar ao aumento da prevalência de hipertensão arterial. Considerando-se o diabetes, sua prevalência não diferiu entre os sexos e não mudou com a idade. Sabe-se que a prevalência desta doença aumenta, principalmente, após os 50 anos ${ }^{16}$. Desta forma, o fato da amostra deste estudo apresentar poucas pessoas com esta idade pode explicar o resultado obtido, além da tendência de aumento da AF total suficiente nesta faixa etária. Em relação à hipercolesterolemia, ela foi maior nos homens e aumentou com a idade. 
As diferenças observadas na prática de $\mathrm{AF}$ entre os gêneros e as idades foram bastante interessantes. Embora estudos ${ }^{10,26,27}$ sugiram que as mulheres sejam menos ativas que os homens, no estudo atual, considerando-se todas as AF, elas tiveram a mesma prevalência de AF total suficiente que os homens, apesar de apresentarem maior prevalência de AF suficiente de deslocamento. Este é um resultado diferente do observado no VIGITEL, no qual o nível de AF de deslocamento das mulheres foi igual aos homens ${ }^{6}$. Novamente, o fato de se estudar uma cidade de pequeno porte, na qual grande parte das mulheres tem atividades domésticas e se movimenta na cidade a pé pode ter influenciado o resultado. É interessante observar que da mesma forma que em diversos outros estudos ${ }^{6,9,10}$, a prevalência de prática de alguma $\mathrm{AF}$ de lazer foi menor nas mulheres que nos homens, sugerindo que mesmo nas cidades de menor porte, as mulheres devem ser estimuladas a praticar AF de lazer.

Com relação à idade, os indivíduos com mais de 36,9 anos tenderam a apresentar menor prevalência de $\mathrm{AF}$ total suficiente, embora a prevalência de $\mathrm{AF}$ suficiente de lazer tenha sido maior nesta faixa etária. Isso sugere uma tendência de aumento da $\mathrm{AF}$ de lazer com o aumento da idade, que já foi identificada em um recente estudo $^{30} \mathrm{e}$, talvez, reflita o resultado de campanhas públicas que tentam incentivar esta prática em adultos para auxiliar no combate às perdas advindas do envelhecimento.

Este estudo apresenta algumas limitações relacionadas a seu delineamento. Por se tratar de uma avaliação transversal não é possível estabelecer relações de causa e efeito, nem a direção de qualquer possível influência. Além disso, como a amostra teve por base pais de crianças do ensino fundamental, a faixa etária dos adultos variou de 24 a 65 anos, de modo que os resultados não devem ser extrapolados para a população adulta em geral de Muzambinho. Porém, já nesta faixa etária, a presença de FRC foi identificada em grande parte da amostra, o que levanta preocupações. $\mathrm{O}$ estudo não utilizou questionários padronizados para a avaliação da $\mathrm{AF}$, nem medida por acelerometria. Utilizou uma entrevista estruturada que avaliou o volume semanal de AF e não considerou a intensidade desta atividade. Este tipo de recurso já foi aplicado em outros estudos ${ }^{13,19}$, mas sua utilização implica em limitações, de modo que os resultados poderiam ser diferentes se outros instrumentos fossem utilizados ou se a intensidade da atividade fosse avaliada, o que precisa ser testado no futuro. Desta forma, é importante ressaltar que os resultados devem ser interpretados à luz da forma como foram avaliados, principalmente na comparação com outros estudos. A reprodutibilidade das medidas não foi testada, porém os equipamentos utilizados são validados e os avaliadores tiveram um extensivo treinamento, o que deve ter minimizado os erros.

Em relação às possíveis aplicações dos resultados, a identificação de alta prevalência de $\mathrm{FRC}$ relacionados à composição corporal concomitante à alta prevalência de altos níveis de $\mathrm{AF}$ numa cidade de pequeno porte demonstra que estas cidades apresentam características diferentes das cidades de médio e grande porte e, portanto, precisam ser analisadas de forma distinta em relação às questões de saúde pública. Além disso, o fato dos altos níveis de AF se deverem, principalmente, à $\mathrm{AF}$ ocupacional e de deslocamento sugere que o incentivo a estes domínios de $\mathrm{AF}$ pode não ser a estratégia ideal para se combater o sobrepeso e a obesidade. Os níveis mais baixos de AF de lazer sugerem que este tipo pode ser mais eficiente neste combate, porém estudos mais específicos devem ser realizados para se verificar a veracidade desta hipótese. 


\section{CONCLUSÃO}

Nos adultos de Muzambinho-MG, as prevalências de FRC e da prática de AF foram altas, sugerindo a coexistência de risco cardiovascular elevado e nível elevado de AF nesta cidade. Os FRC mais prevalentes nos adultos foram os relacionados à gordura corporal (excesso de peso e obesidade central) e a prática de AF elevada se suportou, principalmente, nas elevadas prevalências de AF suficiente nos domínios ocupacional e de deslocamento. As prevalências dos FRC e da prática de $\mathrm{AF}$ nos diferentes domínios variaram com o gênero e com a idade.

\section{Agradecimentos}

Os autores agradecem às Secretarias Municipais de Educação e de Saúde de Muzambinho pelo apoio à execução do estudo; aos professores e dirigentes das instituições de ensino, que participaram deste projeto; aos monitores, que participaram das coletas de dados do projeto; aos adultos, que se voluntariaram para o estudo. Este estudo teve apoio financeiro do CNPq (478249/2007-1), da Pró-Reitoria de Pesquisa da USP (Edital USP-Porto) e da CAPES (Demanda Social).

\section{Contribuição dos autores}

- João Paulo dos Anjos Souza Barbosa - tabulação e análise de dados, bem como escrita do artigo.

- Teresa Bartholomeu, Januária Andrea Souza Rezende, Luciano Basso, Jorge Alberto Oliveira - planejamento, delineamento e organização da coleta e tabulação dos dados, bem como contribuição ao texto do artigo.

- Go Tani, António Prista, José António Ribeiro Maia - Consultores científicos para este artigo e pesquisadores responsáveis e idealizadores do projeto temático em que este artigo se insere.

- Cláudia Lúcia de Moraes Forjaz - idealizadora desta parte específica do estudo temático, responsável geral pelo planejamento, coleta, análise e escrita deste artigo.

\section{REFERÊNCIAS}

1. Ministério da Saúde. Cadernos de informações de saúde 2009. Available from: http://www. datasus.gov.br.

2. Cannon CP. Cardiovascular disease and modifiable cardiometabolic risk factors. Clin Cornerstone. 2007;8(3):11-28.

3. Swift DL, Lavie CJ, Johannsen NM, Arena R, Earnest CP, rsquo, et al. Physical Activity, Cardiorespiratory Fitness, and Exercise Training in Primary and Secondary Coronary Prevention. Circulation Journal. 2013;77(2):281-92.

4. Ministério da Saúde B. Plano de ações estratégicas para enfrentamento das doenças crônicas não transmissíveis (DCNT) no Brasil 2011-2022. 2011(Ministério da Saúde. Secretaria de Vigilância em Saúde. Departamento de Análise de Situação de Saúde):1-160.

5. Ministério da Saúde. Vigilância de fatores de Risco e Proteção para Doenças Crônicas por Inquérito Telefônico. 2009:152.

6. Ministério da Saúde. Vigilância de fatores de Risco e Proteção para Doenças Crônicas por Inquérito Telefônico Brasília: Secretaria da Vigilância em Saúde 2011 [cited 2011]. Available from: http://portalsaude.saude.gov.br/portalsaude/arquivos/pdf/2012/Mai/09/Vigitel_2011_ diabetes_final.pdf.

7. Nunes Filho JR, Debastiani D, Nunes AD, Peres KG. Prevalence of cardiovascular risk factors in adults living in Luzerna, Santa Catarina, in 2006. Arq Bras Cardiol. 2007;89(5):289-93.

8. Oliveira C.M., Pereira A.C., Andrade M., Soler J., Krieger J.E. Heritability of cardiovacular risk factors in a Brazilian population: Baependi Heart Study. BMC Med Genet. 2008;9:32-9.

9. Carvalhaes MAdBL, Moura ECd, Monteiro CA. Prevalência de fatores de risco para doenças crônicas: inquérito populacional mediante entrevistas telefônicas em Botucatu, São Paulo, 2004. Rev Bras de Epidemiologia. 2008;11:14-23. 
10. Doro AR, Gimeno SGA, Hirai AT, Franco LJ, Ferreira SRG. Análise da associação de atividade física à síndrome metabólica em estudo populacional de nipo-brasileiros. Arq Bras Endocrinol \& Metabol. 2006;50:1066-74.

11. Ministério do Planejamento Orçamento e Gestão. Características da população e dos domicílios - Resultados do universo. Rio de Janeiro.2011. p. 270.

12. http://pt.wikipedia.org/wiki/Muzambinho. [acessado em 24/03/2013].

13. Chehuen MdR, Bezerra AIL, Bartholomeu T, Junqueira NO, Rezende JAS, Basso L, et al. Risco cardiovascular e prática de atividade física em crianças e adolescentes de Muzambinho/ MG: influência do gênero e da idade. Rev Bras Med Esporte. 2011;17:232-6.

14. ACSM. ACSM's Guidelines for Exercise Testing and Prescription. Philadelphia: Lippincott Williams and Wilkins; 2010.

15. World Health Organization. Diet, Nutrition and the Prevetion of Chronic Disease. Geneva: WHO, 2003 Contract No.: 916.

16. Diagnosis and classification of diabetes mellitus. Diabetes Care. 2006;29 Suppl 1:S43-8.

17. Sposito AC, Caramelli B, Fonseca FA, Bertolami MC, Afiune Neto A, Souza AD, et al. [IV Brazilian Guideline for Dyslipidemia and Atherosclerosis prevention: Department of Atherosclerosis of Brazilian Society of Cardiology]. Arq Bras Cardiol. 2007;88 Suppl 1:2-19.

18. VI Diretrizes Brasileiras de Hipertensão. Arq Bras de Cardiol. 2010;95:I-III.

19. Forjaz CLM, Bartholomeu T, Rezende JAS, Oliveira JA, Basso L, Tani G, et al. Genetic and environmental influences on blood pressure and physical activity: a study of nuclear families from Muzambinho, Brazil. Braz J Med Biol Res. 2012;45:1269-75.

20. Jousilahti P, Vartiainen E, Tuomilehto J, Puska P. Sex, Age, Cardiovascular Risk Factors, and Coronary Heart Disease: A Prospective Follow-Up Study of 14786 Middle-Aged Men and Women in Finland. Circulation. 1999;99(9):1165-72.

21. Paffenbarger RS, Wing AL, Hyde RT,Jung DL. Physical activity and incidence of hypertension in college alumni. Am J Epidemiol. 1983;117(3):245-57.

22. Jeon CY, Lokken RP, Hu FB, van Dam RM. Physical Activity of Moderate Intensity and Risk of Type 2 Diabetes. Diabetes Care. 2007;30(3):744-52.

23. Matsudo VKR, Matsudo SMM, Araújo TL, Ribeiro MA. Dislipidemias e a promoção da atividade física: uma revisão na persectiva de mensagens de inclusão. Rev Bras Ciên e Mov. 2005;13(2):161-70.

24. Lemmens VEPP, Oenema A, Klepp KI, Henriksen HB, Brug J. A systematic review of the evidence regarding efficacy of obesity prevention interventions among adults. Obesity Reviews. 2008;9(5):446-55.

25. Pinheiro ARdO, Freitas SFTd, Corso ACT. Uma abordagem epidemiológica da obesidade. Rev de Nutrição. 2004;17:523-33.

26. Peixoto MdRG, Monego ET, Alexandre VP, Souza RGMd, Moura ECd. Monitoramento por entrevistas telefônicas de fatores de risco para doenças crônicas: experiência de Goiânia, Goiás, Brasil. Cad Saúde Pública. 2008;24:1323-33.

27. Gomes VB, Siqueira KS, Sichieri R. Atividade física em uma amostra probabilística da população do Município do Rio de Janeiro. Cad Saúde Pública. 2001;17:969-76.

28. Li J, Siegrist J. Physical Activity and Risk of Cardiovascular Disease-A Meta-Analysis of Prospective Cohort Studies. Int J Environ Res Public Health. 2012;9(2):391-407.

29. Matos AC, Ladeia AM. Assessment of cardiovascular risk factors in a rural community in the Brazilian state of Bahia. Arq Bras Cardiol. 2003;81:297-302.

30. Moore SC, Patel AV, Matthews CE, Berrington de Gonzalez A, Park Y, Katki HA, et al. Leisure Time Physical Activity of Moderate to Vigorous Intensity and Mortality: A Large Pooled Cohort Analysis. PLoS Med. 2012;9(11):e1001335.

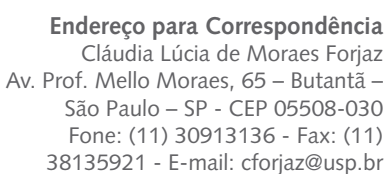

